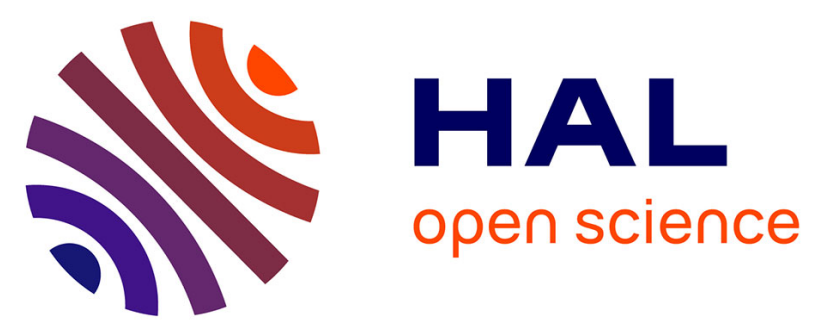

\title{
Effects of age on thermoregulatory responses during cold exposure in a nonhuman primate, Microcebus murinus.
}

Jérémy Terrien, Philippe Zizzari, Marie-Thérèse Bluet-Pajot, Pierre-Yves Henry, Martine Perret, Jacques Epelbaum, Fabienne Aujard

\section{- To cite this version:}

Jérémy Terrien, Philippe Zizzari, Marie-Thérèse Bluet-Pajot, Pierre-Yves Henry, Martine Perret, et al.. Effects of age on thermoregulatory responses during cold exposure in a nonhuman primate, Microcebus murinus.. AJP - Regulatory, Integrative and Comparative Physiology, 2008, 295 (2), pp.R696-703. 10.1152/ajpregu.00629.2007 . hal-00426197

\section{HAL Id: hal-00426197 \\ https://hal.science/hal-00426197}

Submitted on 26 Oct 2009

HAL is a multi-disciplinary open access archive for the deposit and dissemination of scientific research documents, whether they are published or not. The documents may come from teaching and research institutions in France or abroad, or from public or private research centers.
L'archive ouverte pluridisciplinaire HAL, est destinée au dépôt et à la diffusion de documents scientifiques de niveau recherche, publiés ou non, émanant des établissements d'enseignement et de recherche français ou étrangers, des laboratoires publics ou privés. 

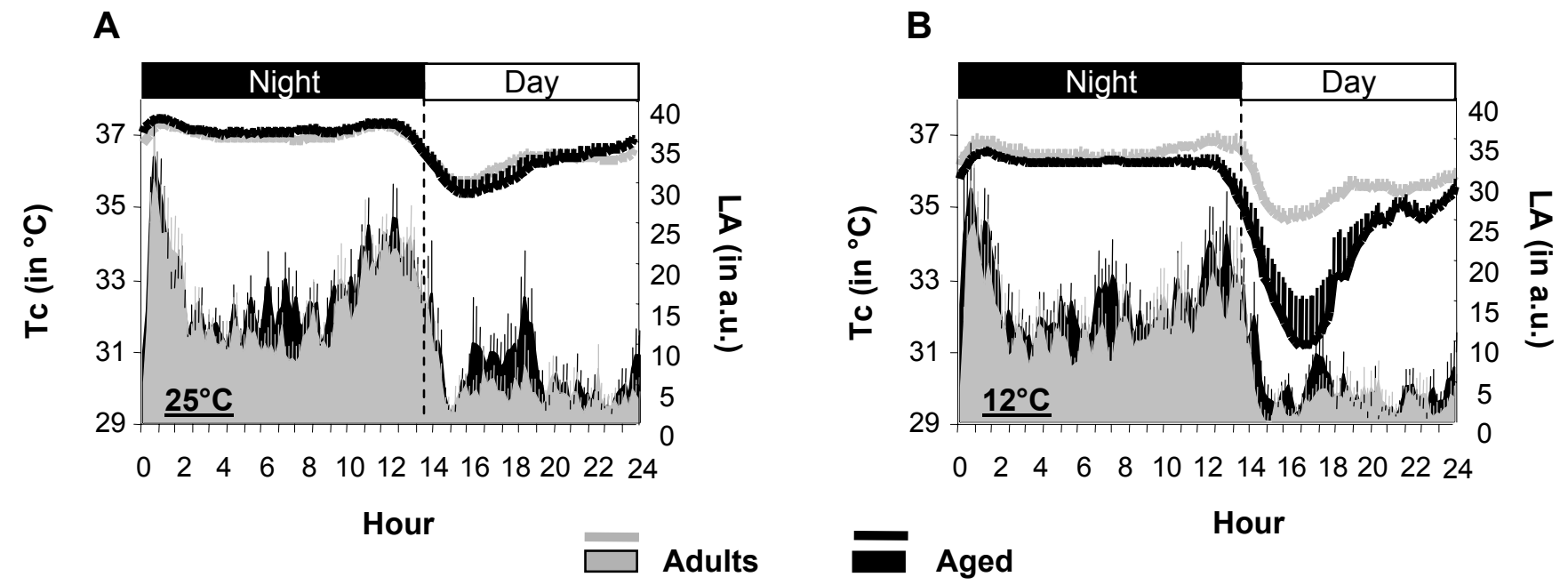

Figure 1. 


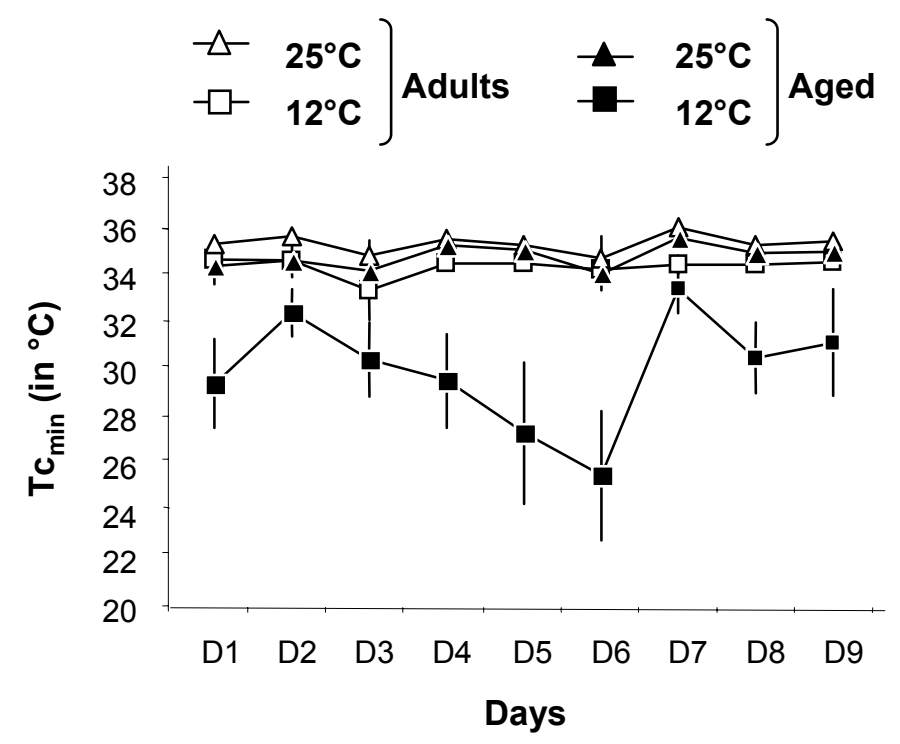

Figure 2. 


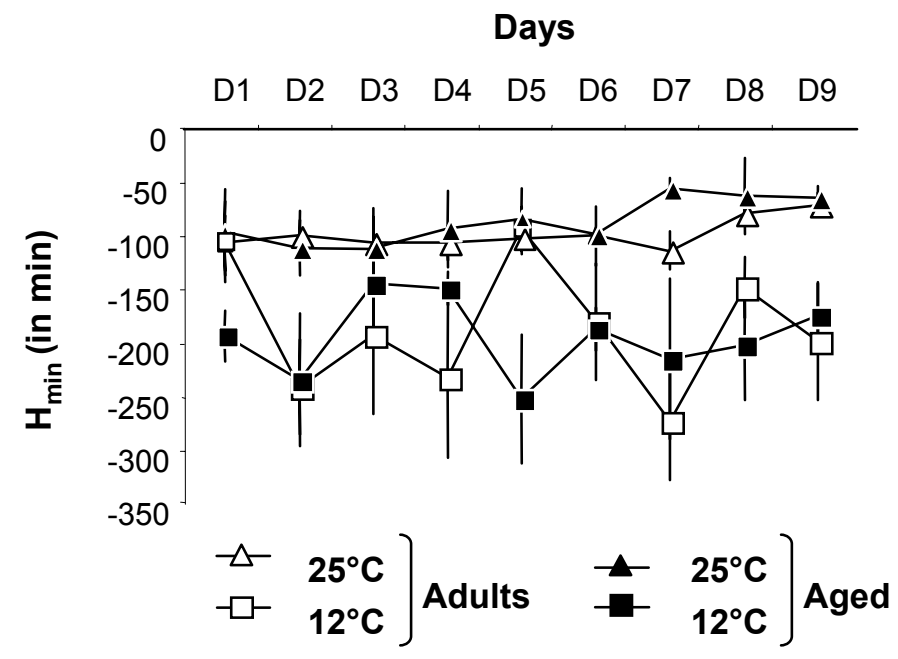

Figure 3. 


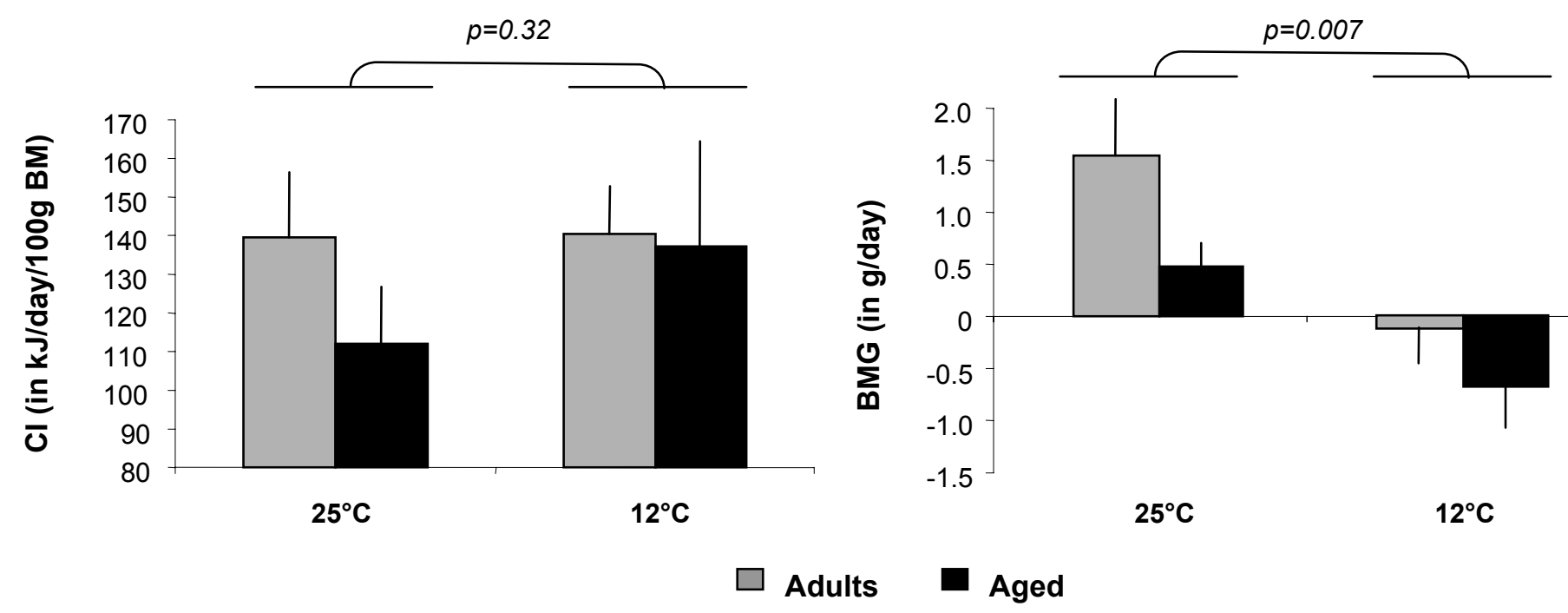

Figure 4. 
Table 1. Rhythm parameters (means values \pm SEM) of core temperature (Tc) and locomotor activity (LA)

determined from profiles of Tc and LA registered in adult and aged mouse lemurs under short photoperiod during the 9 days of exposures to $25^{\circ} \mathrm{C}$ and to $12{ }^{\circ} \mathrm{C}$. Parameters

abbreviations are described in the text.

\begin{tabular}{|c|c|c|c|}
\hline $\mathrm{Ta}$ & Parameters & Adults & Aged \\
\hline \multirow{8}{*}{$25^{\circ} \mathrm{C}$} & $\mathrm{Tc}_{\text {night }}\left({ }^{\circ} \mathrm{C}\right)$ & $37.1 \pm 0.0$ & $37.0 \pm 0.0$ \\
\hline & $\mathrm{Tc}_{\text {day }}\left({ }^{\circ} \mathrm{C}\right)$ & $36.2 \pm 0.1$ & $35.9 \pm 0.1$ \\
\hline & $\mathrm{Tc}_{\min }\left({ }^{\circ} \mathrm{C}\right)$ & $35.4 \pm 0.1$ & $34.8 \pm 0.2$ \\
\hline & $\mathrm{H}_{\text {decr }}(\min )$ & $62 \pm 6$ & $86 \pm 4$ \\
\hline & $\mathbf{H}_{\min }(\min )$ & $-98 \pm 5$ & $-86 \pm 7$ \\
\hline & $F_{\text {torp }}(\%)$ & $3 \pm 3$ & $11 \pm 7$ \\
\hline & LA $_{\text {night }}$ (a.u.) & $1400 \pm 69$ & $1454 \pm 66$ \\
\hline & $\operatorname{LA}_{\text {day }}($ a.u. $)$ & $330 \pm 23$ & $416 \pm 30$ \\
\hline \multirow{8}{*}{$12^{\circ} \mathrm{C}$} & $\mathrm{Tc}_{\text {night }}\left({ }^{\circ} \mathrm{C}\right)$ & $36.7 \pm 0.0$ & $36.1 \pm 0.1$ \\
\hline & $\mathrm{Tc}_{\text {day }}\left({ }^{\circ} \mathrm{C}\right)$ & $35.5 \pm 0.1$ & $32.9 \pm 0.5$ \\
\hline & $\mathrm{Tc}_{\min }\left({ }^{\circ} \mathrm{C}\right)$ & $34.3 \pm 0.1$ & $29.4 \pm 0.9$ \\
\hline & $\mathrm{H}_{\text {decr }}(\min )$ & $48 \pm 5$ & $55 \pm 6$ \\
\hline & $\mathbf{H}_{\min }(\min )$ & $-186 \pm 21$ & $-196 \pm 12$ \\
\hline & $F_{\text {torp }}(\%)$ & $11 \pm 4$ & $67 \pm 13$ \\
\hline & LA $_{\text {night }}$ (a.u.) & $1274 \pm 62$ & $1486 \pm 69$ \\
\hline & LA day (a.u.) & $250 \pm 15$ & $355 \pm 30$ \\
\hline
\end{tabular}


Table 2. Table of $\chi^{2}$ and $p$ values after performing Linear Mixed Effects Models on Tc and LA rhythms testing the effects of age, $\mathrm{Ta}$ and duration of Ta exposures. Parameters abbreviations are described in the text. Tests of effects resulted from comparisons of more complex models with the most parsimonious model (i.e. the model including significant effects only). Effects included in significant interactions were not tested.

\begin{tabular}{|c|c|c|c|c|c|c|c|c|c|c|c|c|c|c|c|c|c|c|c|c|c|}
\hline \multirow{2}{*}{ Effects } & \multicolumn{3}{|c|}{ Tc $c_{\text {night }}$} & \multicolumn{3}{|c|}{$T_{\text {day }}$} & \multicolumn{3}{|c|}{$T c_{\min }$} & \multicolumn{3}{|c|}{$\mathrm{H}_{\text {decr }}$} & \multicolumn{3}{|c|}{$\mathbf{H}_{\min }$} & \multicolumn{3}{|c|}{ LA $_{\text {night }}$} & \multicolumn{3}{|c|}{$\mathrm{LA}_{\text {day }}$} \\
\hline & ddl & $\chi^{2}$ & $p$ & ddl & $\chi^{2}$ & $p$ & ddl & $\chi^{2}$ & $p$ & ddl & $\chi^{2}$ & $p$ & ddl & $\chi^{2}$ & $p$ & ddl & $\chi^{2}$ & $p$ & ddl & $\chi^{2}$ & $p$ \\
\hline Age & & - & & & - & & & - & & 1 & 1.3 & 0.26 & 1 & 0 & 1 & 1 & 0.1 & 0.81 & & - & \\
\hline $\mathrm{Ta}$ & & - & & & - & & & - & & 1 & 3.5 & 0.06 & 1 & 13.4 & 0.00 & 1 & 0.2 & 0.64 & & - & \\
\hline Duration & & - & & & - & & & - & & 8 & 9.2 & 0.33 & 8 & 7.1 & 0.53 & 8 & 34.2 & 0.00 & & - & \\
\hline $\mathrm{Age}^{\star} \mathrm{Ta}$ & & - & & & - & & & - & & & - & & 1 & 0.2 & 0.65 & & - & & & - & \\
\hline Duration*Age & & - & & & - & & & - & & & - & & & - & & 8 & 7.8 & 0.45 & & - & \\
\hline Duration*Ta & & - & & & - & & & - & & & - & & 8 & 7.0 & 0.54 & 8 & 14.5 & 0.07 & & - & \\
\hline Duration*Age ${ }^{*} \mathrm{Ta}$ & 8 & 18.6 & 0.02 & 8 & 19.1 & 0.01 & 8 & 18.7 & 0.02 & & - & & & - & & & - & & 8 & 15.9 & 0.04 \\
\hline
\end{tabular}


Table 3. IGF-1 blood levels (mean + SEM, $\mathrm{ng} / \mathrm{ml}$ in $100 \mathrm{~g} \mathrm{BM}$ ) in adult and aged mouse lemurs at $25^{\circ} \mathrm{C}$, after 2 days and after 9 days of exposure to $12{ }^{\circ} \mathrm{C}$. Linear Mixed Effects Models were performed to test the effects of age and ambient temperature (Ta) on the modulation of IGF-1 blood levels..

\begin{tabular}{|c|c|c|c|c|c|c|}
\cline { 2 - 6 } \multicolumn{1}{c|}{} & \multirow{2}{*}{$\mathbf{2 5} 5^{\circ} \mathbf{C}$} & \multicolumn{2}{c|}{$12^{\circ} \mathbf{C}$} & \multicolumn{2}{c|}{ LME } \\
\cline { 3 - 7 } \multicolumn{1}{c|}{} & & Day 2 & Day 9 & age effect & Ta effect & age*Ta effect \\
\hline Adults & $592 \pm 99$ & $444 \pm 42$ & $537 \pm 48$ & \multirow{2}{*}{$\chi_{(1)}^{2}=1.25 ; p=0.26$} & $\chi_{(2)}^{2}=8.04 ; p=0.02$ & $\chi^{2}{ }_{(2)}=0.04 ; p=0.98$ \\
\hline Aged & $721 \pm 144$ & $505 \pm 122$ & $671 \pm 125$ & & \\
\hline
\end{tabular}

